\title{
Fenômeno de Fluxo Lento Coronariano - Adicionando Fibrose Miocárdica à Equação
}

\author{
Coronary Slow Flow Phenomenon - Adding Myocardial Fibrosis to the Equation
}

\author{
Filipe Penna de Carvalho ${ }^{1,2}$ e Clério Francisco de Azevedo ${ }^{1,3}$ (ำ \\ Diagnósticos da América SA, ${ }^{1}$ Rio de Janeiro, RJ - Brasil \\ Américas Serviços Médicos, ${ }^{2}$ Rio de Janeiro, RJ - Brasil \\ Duke University Hospital - Medicine/Cardiology, ${ }^{3}$ Durham, North Carolina - EUA \\ Minieditorial referente ao artigo: Determinação do Tecido Cicatricial do Miocárdio no Fenômeno de Fluxo Coronário Lento e a Relação entre \\ a Quantidade de Tecido Cicatricial e o Nt-ProBNP
}

Inicialmente descrito há mais de 40 anos por Tambe et al., ${ }^{1}$ o fenômeno de fluxo lento coronariano (FLC) é caracterizado por retardo da progressão do meio de contraste na ausência de doença epicárdica coronariana obstrutiva durante angiografia coronária invasiva $(\mathrm{ACl}){ }^{2} \mathrm{O}$ FLC afeta tipicamente jovens fumantes do sexo masculino, que frequentemente apresentam síndrome coronariana aguda (SCA) ou angina de repouso refratária recorrente que requer internação. ${ }^{2-4}$ Além disso, arritmias potencialmente fatais e morte súbita cardíaca também foram associadas ao FLC. ${ }^{5}$

Apesar do aumento da conscientização e da pesquisa, o FLC permanece uma condição difícil de descrever e pouco compreendida, com muitos mecanismos patogênicos propostos, incluindo disfunção endotelial, vasomotora e microvascular. ${ }^{2,6}$ De fato, uma regulação anormal do tônus microvascular que ocorre apenas durante condições de repouso, enquanto a reserva de fluxo coronariano está dentro da faixa normal, foi descrita no FLC. ${ }^{7}$

Nessa edição dos Arquivos Brasileiros de Cardiologia, Candemir et al., ${ }^{8}$ dão uma contribuição importante a esse campo do conhecimento. Os autores estudaram 35 pacientes com dor torácica encaminhados para uma angiografia coronária invasiva $(\mathrm{ACl})$ diagnóstica. Todos tinham níveis negativos de troponina e nenhuma evidência de isquemia no teste ergométrico. Foi feita uma comparação entre os pacientes que apresentaram FLC na artéria descendente anterior esquerda $(n=19)$ e os controles pareados com artérias coronárias normais e sem anormalidades do fluxo coronariano $(n=16)$. Eles procuraram investigar se as cicatrizes no miocárdio identificadas por ressonância magnética cardíaca (RMC) e / ou se os níveis do fragmento $\mathrm{N}$-terminal do peptídeo natriurético tipo B (NT-ProBNP) eram

\section{Palavras-chave}

Insuficiência Cardíaca; Reserva Fracionada de Fluxo Miocárdio; Cicatriz Hipertrófica; Prognóstico; Peptídeo Natriurético Tipo C; Fibrose Endomiocárdica; Espectroscopia de Ressonância Magnética/métodos.

Correspondência: Filipe P. Carvalho •

Centro Integrado de Diagnóstico do Leblon, CDPI Cardiologia - $2^{\circ}$ andar -

Av. Ataulfo de Paiva, 669. CEP 22440-032, Rio de Janeiro, RJ - Brasil E-mail: filipepenna@me.com

DOI: https://doi.org/10.36660/abc.20200187 mais frequentes no grupo com FLC. É importante ressaltar que, pelo que sabemos, este foi o primeiro estudo a utilizar a RMC para avaliar a presença de fibrose miocárdica na população com FLC.

Vale ressaltar que a RMC utilizando a técnica de realce tardio é agora uma ferramenta amplamente disponível e poderosa que permite a identificação e quantificação precisa da fibrose miocárdica, com vários estudos demonstrando sua utilidade no diagnóstico e prognóstico de cardiomiopatias tanto isquêmicas quanto não isquêmicas. ${ }^{9-15}$ Curiosamente, os autores demonstram que o realce tardio estava presente em até 52,5\% $(n=10)$ dos pacientes com FLC, em oposição a nenhum no grupo controle. Os autores concluíram que o FLC pode resultar em alterações irreversíveis no tecido miocárdico.

Entretanto, não acreditamos que os dados apresentados possam ser utilizados para estabelecer causalidade entre FLC e fibrose miocárdica. Por exemplo, em um subgrupo de pacientes $(n=3)$, a lesão miocárdica foi observada nas paredes inferior e ínfero-lateral, e não no território típico da artéria coronária descendente anterior esquerda, onde estava presente o FLC. Além disso, os autores não descrevem se o padrão de realce tardio observado em seu estudo era predominantemente isquêmico (por exemplo, subendocárdico ou transmural) ou não-isquêmico (mesocárdico ou epicárdico). Mais importante ainda, como os autores apontam durante a discussão, eles realizaram um estudo transversal e, portanto, nenhuma relação temporal pode ser estabelecida entre o FLC e a fibrose miocárdica. Uma das muitas explicações possíveis é que esses pacientes com fibrose miocárdica na RMC podem ter apresentado anteriormente um infarto do miocárdio com artérias coronárias normais (MINOCA, do inglês myocardial infarction with normal coronary arteries) e o FLC é apenas uma consequência desse evento anterior. Embora os autores tenham encontrado uma associação entre o FLC e a fibrose miocárdica, acreditamos que são necessários mais estudos para determinar se existe uma relação causal entre eles.

Curiosamente, níveis mais altos de NT-pro-BNP, um conhecido marcador prognóstico na $\mathrm{SCA},{ }^{16}$ também foram observados em pacientes com FLC quando as cicatrizes miocárdicas foram detectadas por RMC, em comparação com o FLC sem evidência de fibrose na RMC (NT-pro- BNP $=147,10 \mathrm{pg} / \mathrm{mL}$ vs. 28,0 pg / mL, $\mathrm{p}=0,03$ ).

É importante ressaltar que, em um estudo publicado anteriormente por Yurtdaş et al., ${ }^{6}$ demonstrou-se que níveis 
elevados de NT-pro-BNP se correlacionaram com angina e infradesnivelamento do segmento ST em pacientes com FLC durante testes em esteira. No entanto, nenhuma anormalidade foi observada durante o teste ergométrico em nenhum paciente deste estudo. Novamente, embora os autores demonstrem uma associação de NT-pro-BNP com FLC e fibrose miocárdica, acreditamos que nenhuma relação causal definitiva possa ser estabelecida com base nos dados apresentados.

Em geral, este é um trabalho interessante de Candemir et al., ${ }^{8}$ utilizando a RMC para estudar uma condição ainda obscura. Achamos muito interessante que a RMC tenha permitido a detecção de fibrose miocárdica em um subgrupo de pacientes com FLC sem história prévia de infarto do miocárdio. A identificação de cicatrizes miocárdicas utilizando imagens de realce tardio é uma poderosa ferramenta prognóstica em múltiplas cardiomiopatias, isquêmicas e não isquêmicas. Embora pequeno em tamanho, este estudo de Candemir et al., ${ }^{8}$ abre novas possibilidades de pesquisa para responder se há causalidade na associação entre FLC e fibrose miocárdica e se a presença de fibrose miocárdica nesses pacientes tem alguma implicação prognóstica ou se, por exemplo, está associada a uma maior probabilidade de arritmias malignas. Por outro lado, pesquisas futuras utilizando novas técnicas de RMC para caracterização de tecidos, incluindo o mapeamento de T1 e T2, também podem ajudar a esclarecer essa condição ainda pouco compreendida.

\section{Referências}

1. Tambe AA, Demany MA, Zimmerman HA, Mascarenhas E. Angina pectoris and slow flow velocity of dye in coronary arteries-A new angiographic finding. Am Heart J. 1972;84(1):66-71.

2. Wang X, Nie S-P. The coronary slow flow phenomenon: characteristics, mechanisms and implications. Cardiovasc Diagn Ther. 2011;1(1):37-43.

3. Fineschi M, Gori T. Coronary Slow-Flow Phenomenon or Syndrome Y. J Am Coll Cardiol. 2010;56(3):239-40.

4. Beltrame JF, Limaye SB, Horowitz JD. The Coronary Slow Flow Phenomenon - A New Coronary Microvascular Disorder. Cardiology. 2002;97(4):197202.

5. Saya S, Hennebry TA, Lozano P, Lazzara R, Schechter E. Coronary Slow Flow Phenomenon and Risk for Sudden Cardiac Death Due to Ventricular Arrhythmias: A Case Report and Review of Literature. Clin Cardiol. 2008;31(8):352-5.

6. Yurtdaş M, Özcan IT, Çamsari A, Cice KD, Tamer L, Cin VC. NT-pro-BNP levels and their response to exercise in patients with slow coronary flow. Arq Bras Cardiol. 2012;99(6):1115-22.

7. Fineschi M, Bravi A, Gori T. The "slow coronary flow" phenomenon: Evidence of preserved coronary flow reserve despite increased resting microvascular resistances. Int J Cardiol. 2008;127(3):358-61.

8. Candemir M, Sahinarslan A, Yazol M, Oner YA, Boyaci B. Determinação do tecido cicatricial do miocádio no fenômeno de fluxo coronário lento e a relação entre quantidade de tecido cicatricial e o Nt-Pro Bnp. Arq Bras Cardiol. 2020; 114(3):540-551

9. Sara L, Szarf G, Tachibana A,Shiozaki AA, Villa AV, Oliveira AC, et al. II Diretriz de Ressonância Magnética e Tomografia computadorizada
Cardiovascular da Sociedade Brasileira de Cardiologia e do Colégio Brasileiro de Radiologia. Arq Bras Cardiol. 2014;103(6):1-86.

10. Wu KC, Kim RJ, Bluemke D, Rochitte CE, Zerhouni EA, Becker LC, et al. Quantification and time course of microvascular obstruction by contrast-enhanced echocardiography and magnetic resonance imaging following acute myocardial infarction and reperfusion. J Am Coll Cardiol. 1998;32(6):1756-64

11. Kim HW, Farzaneh-Far A, Kim RJ. Cardiovascular Magnetic Resonance in Patients With Myocardial Infarction. Current and Emerging Applications. J Am Coll Cardiol. 2009;55(1):1-16

12. Azevedo CF, Nigri M, Higuchi ML, Pomerantzeff PMA, Spina G, Sampaio $\mathrm{R}$, et al. Prognostic significance of myocardial fibrosis quantification by histopathology and magnetic resonance imaging in patients with severe aortic valve disease. J Am Coll Cardiol. 2010;56(4):278-87.

13. Azevedo Filho CF de, Hadlich M, Petriz JLF, Mendonça LA, Moll Filho JN Rochitte CE. Quantificação da massa infartada do ventrículo esquerdo pela ressonância magnética cardíaca: comparação entre a planimetria e o método de escore visual semi-quantitativo. Arq Bras Cardiol. 2004;83(2):111-7.

14. Carvalho FP de, Erthal F, Azevedo CF. The Role of Cardiac MR Imaging in the Assessment of Patients with Cardiac Amyloidosis. Magn Reson Imaging Clin NAm. 2019;27(3):453-63.

15. de Carvalho FP, Azevedo CF. Comprehensive Assessment of Endomyocardial Fibrosis with Cardiac MRI: Morphology, Function, and Tissue Characterization. RadioGraphics. Jan. 2020:190148.

16. Haaf P, Balmelli C, Reichlin T, Twerenbold R, Reiter M, Meissner J,et al. N-terminal Pro B-type Natriuretic Peptide in the Early Evaluation of Suspected Acute Myocardial Infarction. Am J Med. 2011;124(8):731-9. 